\title{
PP-attachment disambiguation using large context
}

\author{
Marian Olteanu and Dan Moldovan \\ Human Language Technology Research Institute \\ The University of Texas at Dallas \\ Richardson, TX 75080 \\ marian@hlt.utdallas.edu \\ moldovan@utdallas.edu
}

\begin{abstract}
Prepositional Phrase-attachment is a common source of ambiguity in natural language. The previous approaches use limited information to solve the ambiguity - four lexical heads - although humans disambiguate much better when the full sentence is available. We propose to solve the PP-attachment ambiguity with a Support Vector Machines learning model that uses complex syntactic and semantic features as well as unsupervised information obtained from the World Wide Web. The system was tested on several datasets obtaining an accuracy of $93.62 \%$ on a Penn Treebank-II dataset; $91.79 \%$ on a FrameNet dataset when no manuallyannotated semantic information is provided and $92.85 \%$ when semantic information is provided.
\end{abstract}

\section{Problem description}

\subsection{PP-attachment ambiguity problem}

Prepositional Phrase-attachment is a source of ambiguity in natural language that generates a significant number of errors in syntactic parsing. For example the sentence "I saw yesterday the man in the park with a telescope" has 5 different semantic interpretations based on the way the prepositional phrases "in the park" and "with the telescope" are attached: I saw yesterday [the man [in the park [with a telescope]]]; I saw yesterday [the man [in the park] [with a telescope]]; I saw yesterday [the man [in the park]] [with a telescope]; I saw yesterday [the man] [in the park [with a telescope]] and I saw yesterday [the man] [in the park] [with a telescope].

The problem can be viewed as a decision of attaching a prepositional phrase (PP) to one of the preceding head nouns or verbs. The ambiguity expressed by the number of potential parse trees generated by Context-Free Grammars increases exponentially with the number of PPs. For a PP that follows the object of a verb there are 2 parse trees, for a chain of 2, 3, 4 and 5 PPs there are respectively 5, 14, 42 and 132 parse trees. Usually the average number of consecutive PPs in a sentence increases linearly with the length of the sentence.

Lexical and syntactic information alone is not sufficient to resolve the PP-attachment problem; often semantic and/or contextual information is necessary. For example, in "I ate a pizza with anchovies", "with anchovies" attaches to the noun "pizza", where as in "I ate a pizza with friends.", "with friends" attaches to the verb "eat" - example found in (McLauchlan, 2001). There are instances of PP-attachment, like the one in "I saw the car in the picture" that can be disambiguated only by using contextual discourse information.

Usually, people don't have much trouble in finding the right way to attach PPs. But if one limits the information used for disambiguation of the PPattachment to include only the verb, the noun representing its object, the preposition and the main noun in the PP, the accuracy for human decision degrades from $93.2 \%$ to $88.2 \%$ (Ratnaparkhi et al., 1994) on a dataset extracted from Penn Treebank (Marcus et 
al., 1993).

\subsection{Motivation}

Syntactic parsing is essential for many natural language applications such as Machine Translation, Question Answering, Information Extraction, Information Retrieval, Automatic Speech Recognition. Since parsing occurs early in the chain of NLP processing steps it has a large impact on the overall system performance.

\section{Approach}

Our approach to solve the PP-attachment ambiguity is based on a Support Vector Machines learner (Cortes and Vapnik, 1995). The feature set contains complex information extracted automatically from candidate syntax trees generated by parsing (Charniak, 2000), trees that will be improved by more accurate PP-attachment decisions. Some of these features were proven efficient for semantic information labeling (Gildea and Jurafsky, 2002). The feature set also includes unsupervised information obtained from a very large corpus (World Wide Web). Features containing manually annotated semantic information about the verb and about the objects of the verb have also been used. We adopted the standard approach to distinguish between verb and noun attachment; thus the classifier has to choose between two classes: V when the prepositional phrase is attached to the verb and $\mathrm{N}$ when the prepositional phrase is attached to the preceding head noun.

\section{Data}

To be able to extract the required features from a dataset instance, one must identify the verb, the phrase identifying the object of the verb that precedes the prepositional phrase in question $(n p 1)$ which usually is part of the predicate-argument structure of the verb, its head noun, the prepositional phrase ( $n p 2)$, its preposition and its head noun (the second most important word in the PP).

We have adopted the notation from (Collins and Brooks, 1995), where $v$ is the verb, $n_{1}$ is the head noun of object phrase, $p$ is the preposition and $n_{2}$ is the head noun of the prepositional phrase.

Compared to our datasets, Ratnaparkhi's dataset (Ratnaparkhi et al., 1994) contains only the lexical heads $v, n_{1}, p$ and $n_{2}$. Thus, our methodology cannot be applied to Ratnaparkhi's dataset (RRR).

In our experiments we used two datasets:
- FN - extracted from FrameNet II 1.1 (Baker et al., 1998)

- TB2 - extracted from Penn Treebank-II

Table 1 presents the datasets ${ }^{1}$. The creation of the datasets is described in details in (Olteanu, 2004).

\section{Features}

The experiments described in this paper use a set of discrete (alphanumeric) and continuous (numeric) features. All features are fully deterministic, except the features count-ratio and pp-count that are based on information provided by an external resource - Google search engine (http: //www.google. $\mathrm{com})$.

In describing the features, we will use the Penn Treebank-II parse tree associated with the sentence "The Lorillard spokeswoman said asbestos was used in "very modest amounts" in making paper for the filters in the early 1950s and replaced with a different type of filter in 1956".

Table 2 describes the features and the origin of each feature. The preposition is the feature with the most discriminative power, because of preferences of particular prepositions to attach to verbs or nouns. Table 3 shows the distribution of top 10 most frequently used prepositions in the $\mathrm{FN}$ and TB2 datasets.

The features were carefully designed so that, when they are extracted from gold parse trees, they don't provide more information useful for disambiguation than when they are automatically generated using a parser. This claim is validated by the experimental results that show a strong correlation between the results on the two datasets - one based on automatically generated parse trees (FN) and one based on gold parse trees (TB2).

Next, we describe in further detail the features presented in Table 2.

$\mathbf{v}$-frame represents the frame of the verb - the frame to which the verb belongs, as it is present in FrameNet (manually annotated). We used this feature because the frame of the verb describes very well the semantic behavior of the verb including the predicate-argument structure of the verb, which entails the affinity of the verb for certain prepositions.

\footnotetext{
${ }^{1}$ The datasets are available at http: / / www . utdallas. edu/ mgo0 $31000 / \mathrm{ppa} /$
} 


\begin{tabular}{|l|l|l|}
\hline & FN & TB2 \\
\hline \hline Source & $\begin{array}{l}\text { FrameNet annotation samples (British National } \\
\text { Corpus) }\end{array}$ & $\begin{array}{l}\text { Penn Treebank-II } \\
\text { (WSJ articles) }\end{array}$ \\
\hline $\begin{array}{l}\text { Instance identifica- } \\
\text { tion }\end{array}$ & Semantic-centered (related to Frame Elements) & $\begin{array}{l}\text { Syntactic-centered (related to the structure of the } \\
\text { parse tree) }\end{array}$ \\
\hline Parse trees & Automatically generated (Charniak) & Gold standard \\
\hline Total size & 27,421 instances & 60,699 instances \\
\hline Distribution statistics & $\begin{array}{l}70.28 \% \text { ambiguous verb attachments } \\
2.36: 1 \text { v-attch:n-attch }\end{array}$ & $\begin{array}{l}35.71 \% \text { ambiguous verb attachments } \\
1: 1.8 \text { v-attch:n-attch }\end{array}$ \\
\hline Training / test sets & $90 \%-10 \%$ - homogenously distributed (one in every 10 instances is selected for the test set) \\
\hline Location of PP & Both before and after verb & Only after verb \\
\hline Other properties & - Partial identification of ambiguous PP- & \\
& $\begin{array}{l}\text { attachment instances in the corpus, derived from } \\
\text { manual annotation of FEs (Olteanu, 2004) }\end{array}$ & \\
& - Semantic information readily available & \\
\hline
\end{tabular}

Table 1: The datasets and their characteristics

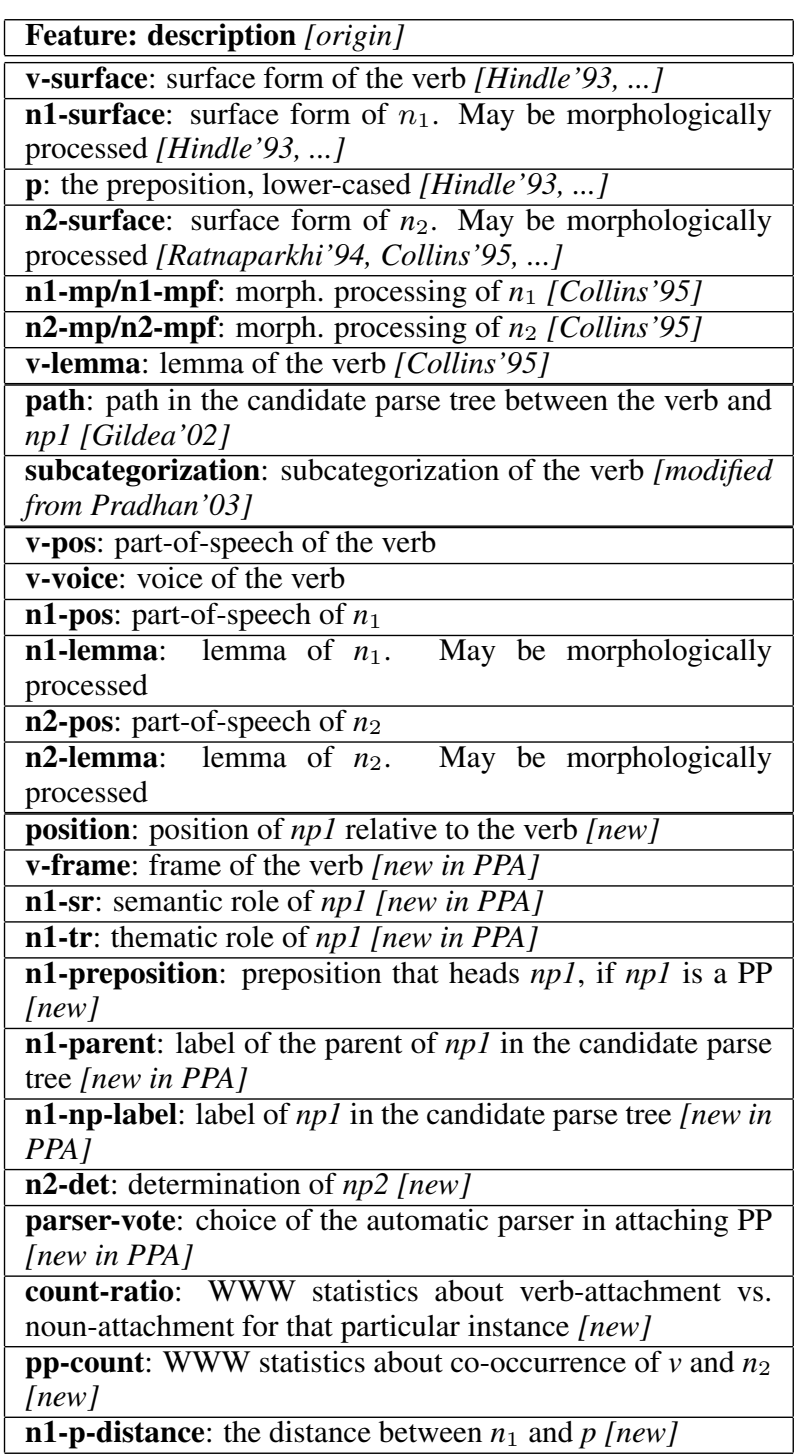

Table 2: Features

\begin{tabular}{|l||r|r||r|r|}
\hline Prep. & $\begin{array}{r}\% \text { of } \\
\text { FN }\end{array}$ & $\begin{array}{r}\% \text { v-att } \\
\text { FN }\end{array}$ & $\begin{array}{r}\text { \% of } \\
\text { TB2 }\end{array}$ & $\begin{array}{r}\text { \% v-att } \\
\text { TB2 }\end{array}$ \\
\hline of & $13.47 \%$ & $6.17 \%$ & $30.14 \%$ & $2.74 \%$ \\
\hline to & $13.27 \%$ & $80.14 \%$ & $9.55 \%$ & $60.49 \%$ \\
\hline in & $12.42 \%$ & $73.64 \%$ & $16.94 \%$ & $42.58 \%$ \\
\hline for & $6.87 \%$ & $82.44 \%$ & $8.95 \%$ & $39.72 \%$ \\
\hline on & $6.21 \%$ & $75.51 \%$ & $5.16 \%$ & $47.73 \%$ \\
\hline with & $6.17 \%$ & $86.30 \%$ & $3.79 \%$ & $46.92 \%$ \\
\hline from & $5.37 \%$ & $75.90 \%$ & $5.76 \%$ & $52.76 \%$ \\
\hline at & $4.09 \%$ & $76.63 \%$ & $3.21 \%$ & $66.02 \%$ \\
\hline as & $3.95 \%$ & $86.51 \%$ & $2.49 \%$ & $51.69 \%$ \\
\hline by & $3.53 \%$ & $88.02 \%$ & $3.27 \%$ & $68.11 \%$ \\
\hline
\end{tabular}

Table 3: Distribution of the first 10 most-frequent prepositions in the FN and TB2 datasets

n1-sr represents the semantic role of the object phrase npl - the label attached to the Frame Element (manual semantic annotation that can be found in FrameNet). This feature was introduced because of the relation between the underlying meaning of $n p l$ and its semantic role.

n1-tr represents the thematic role of the object phrase $n p 1$ - a coarse-grained role based on the label attached to the Frame Element (manual semantic annotation that can be found in FrameNet). It was introduced to reduce data sparseness for the n1-sr feature. The conversion from fine-grained semantic role to coarse-grained semantic role is done automatically using a table that maps a pair of a framelevel semantic role (FE label) and a frame to a thematic role.

subcategorization contains a semi-lexicalized description of the structure of the verb phrase. A subcategorization frame is closely related to the 
predicate argument structure and to the underlying meaning of the verb. It contains an ordered set of all the phrase labels that are siblings of the verb, plus a marker for the verb. If the child phrase of the verb is a PP, then the label will also contain the preposition (the headword of the PP). This feature is a modified form of the sub-categorization feature described in (Pradhan et al., 2003): the differences in various part-of-speeches for the verb were ignored and the preposition that heads a prepositional phrase is also attached to the label. Therefore, for the sentence "The stock declined in June by 4\%", the value for this feature is *-PPin-PPby.

In the TB2 dataset the parse trees are gold standard (contain the expected output value for PPambiguity resolution). In the case of a verb attachment, if the selected PP is a child of the selected VP, then by applying the algorithm, the value of the feature will contain the PP label plus the preposition. This clearly is a clue for the learner that the instance is a verb attachment. To overcome this problem for datasets based on gold-standard parse trees, when computing the value of the subcategorization feature the selected PP will not be used. Figure 1 shows the subcategorization for the phrase "replaced with a different type of filter in 1956".

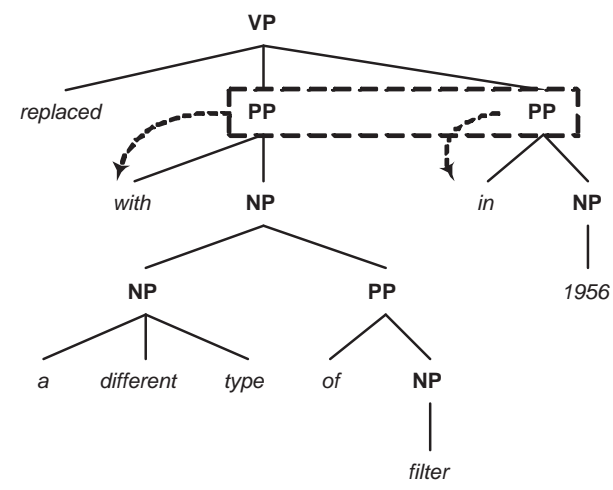

Figure 1: Subcategorization feature: *-PPin-PPby

path expresses the syntactic relation between the verb $v$ and the object phrase $n p l$. Its purpose is to describe the syntactic relation of $n p 1$ to the rest of the clause by the syntactic relation of $n p l$ with the head of the clause $-v$. We adopted this feature from (Gildea and Jurafsky, 2002). path describes the chain of labels in the tree from $v$ to $n p l$, includ- ing the label of $v$ and $n p l$. Ascending movements and descending movements are depicted separately. We used two variants of this feature to determine the optimum version for our problem - one with full POS of the verb and one with POS reduced to "VB". The experiments proved that the second variant provides a better performance. Figure 2 depicts the path between "replaced" and "a different type of filter": $\mathrm{VBN} \uparrow \mathrm{VP} \downarrow \mathrm{PP} \downarrow \mathrm{NP}$ or $\mathrm{VB} \uparrow \mathrm{VP} \downarrow \mathrm{PP} \downarrow N \mathrm{~N}$.

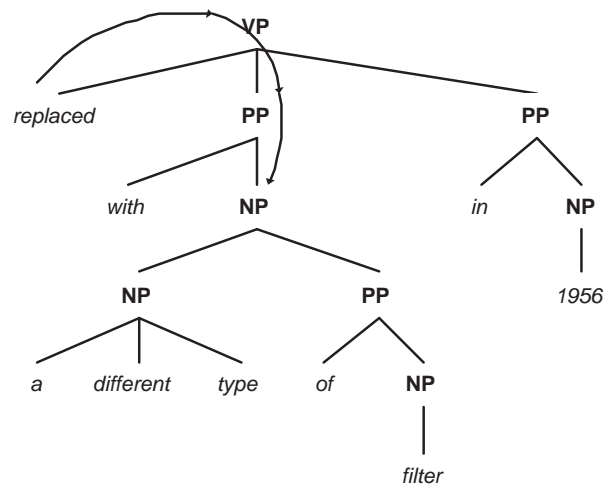

Figure 2: Example of a path feature

position indicates the position of the $n_{1}-p-n_{2}$ construction relative to the verb, i.e. whether the prepositional phrase in question lies before the verb or after the verb in the sentence. Position is very important in deciding the type of attachment, considering the totally different distribution of PPs constructions preceding the verb and PPs constructions following the verb.

Morphological processing applied to $n_{1}$ and $n_{2}$ was inspired by the algorithm described in (Collins and Brooks, 1995). We analyzed the impact of different levels of morphological processing by using two types: partial morphological processing (only numbers and years are converted) - identified by adding - $\mathbf{m p}$ as a suffix to the name of this feature and full morphological processing (numbers, years and capitalized names) - identified by adding - $\mathbf{m p f}$ as a suffix to the name of this feature. The purpose of morphological processing is data sparseness reduction by clustering similar values for this feature.

n1-parent represents the phrase label of the parent of np1 and it cannot be used on gold parse trees (TB2 dataset) because it will provide a clue about the correct attachment type. 
n2-det is called the determination of the prepositional phrase $n p 2$. This novel feature tells if $n_{2}$ is preceded in $n p 2$ by a possessive pronoun or by a determiner. This is used to differentiate between "buy books for children" (which is probably a noun attachment) and "buy books for her children" (which very probably is a verb attachment).

parser-vote feature represents the choice of the parser (Charniak's parser) in the PP-attachment resolution. It cannot be used with gold-standard parse trees because it will provide the right answer.

count-ratio represents the estimated ratio between the frequency of an unambiguous verb attachment construction based on $v, p$ and $n_{2}$ and the frequency of a probably unambiguous noun attachment construction based on $n_{1}, p$ and $n_{2}$ in a very large corpus. A very large corpus is required to overcome the data sparseness inherent for complex constructions like those described above.

We chose the World Wide Web as a corpus and Google as a query interface (see (Olteanu, 2004) for details).

Let's consider the estimated frequency of unambiguous verb-attachments and respectively nounattachments defined as:

$$
\begin{aligned}
f_{v} & =\frac{c_{v-p-n 2}}{c_{v} \cdot c_{p-n 2}} \\
f_{n} & =\frac{c_{n 1-p-n 2}}{c_{n 1} \cdot c_{p-n 2}}
\end{aligned}
$$

where:

- $c_{v-p-n 2}$ is the number of occurrences of the phrase " $v p n_{2}$ ", " $v p * n_{2}$ " (where * symbolizes any word), "v-lemma p $n_{2}$ " or " $v$-lemma $p * n_{2}$ " in World Wide Web, as reported by Google

- $c_{v}$ is the number of occurrences of the word " $v$ " or " $v$-lemma" in WWW

- $c_{p-n 2}$ is the number of occurrences of the phrase " $p n_{2}$ " or " $p * n_{2}$ " in WWW

- $c_{n 1-p-n 2}$ is the number of occurrences of the phrase " $n_{1} p n_{2}$ " or " $v p * n_{2}$ " in WWW

- $c_{n 1}$ is the number of occurrences of the word " $n_{1}$ " in WWW

The value for this feature is:

$$
\text { count }- \text { ratio }=\log _{10} \frac{f_{v}}{f_{n}}=\log _{10} \frac{c_{v-p-n 2} \cdot c_{n 1}}{c_{n 1-p-n 2} \cdot c_{v}}
$$

We chose logarithmic values for this feature because experiments showed that logarithmic values provide a higher accuracy than linear values. Also, by experimentation we concluded that value bounding is helpful, and the feature was bounded to values between -3 and 3 on the logarithmic scale, unless specified otherwise in the experiment description.

This feature resembles the approach adopted in (Volk, 2001).

pp-count depicts the estimated count of occurrences in World Wide Web of the prepositional phrases based on $p$ and $n_{2}$. The count is estimated by $c_{p-n 2}$. Therefore pp-count $=\log _{10}\left(c_{p-n 2}+\right.$ $\left.c_{p-*-n 2}\right)$.

n1-p-distance depicts the distance (in tokens) between $n_{1}$ and $p$. Let $d_{n 1-p}$ be the distance between $n_{1}$ and $p(d=1$ if there is no other token between $n_{1}$ and $p$ ). Thus n1-p-distance $=$ $\log _{10}\left(1+\log _{10} d_{n 1-p}\right)$.

\section{Learning model and procedure}

We used in our experiments a Support Vector Machines learner with Radial Basis Function kernel as implemented in the LIBSVM toolkit (http://www.csie.ntu.edu.tw/ ${ }^{c j l i n /}$ libsvm/).

We converted the feature tuples (containing discrete alphanumeric and continuous values) to multidimensional vectors using the following procedure:

- Discrete features: assign to each possible value of each feature a dimension in the vector space, and to each feature value in each training or test example put 1 in the dimension corresponding to the feature value and 0 in all other dimensions associated with that feature.

- Continuous features: assign a dimension and put the scaled value in the multi-dimensional vector (all examples in training data will span between 0 and 1 for that particular dimension).

SVM training was preceded by finding the optimal $\gamma$ and $C$ parameters required for training using 2-fold cross validation, which was found to be superior in model accuracy and training time over higher folds cross-validations (Olteanu, 2004).

The criterion for selecting the best set of features was the accuracy on the cross-validation. Thus, the development of the models was performed entirely 
on the training set, which acted also as a development set. We later computed the accuracy on the test set on some representative models.

\section{Experiments, results and analysis}

For each dataset, we conducted experiments to determine an efficient combination of features and the accuracy on test data for the best combination of features. We also run the experimental procedure on the original Ratnaparkhi's dataset in order to compare SVM with other machine learning techniques applied to PP-attachment problem. Table 4 summarizes the experiments performed on all datasets.

\begin{tabular}{|l|r|r|}
\hline Experiment & $\begin{array}{r}\text { \% on dev } \\
\text { / x-val }\end{array}$ & \% on test \\
\hline \hline FN-basic-flw & 86.25 & 86.44 \\
\hline FN-lex-syn-flw & 88.55 & 89.61 \\
\hline FN-best-no-sem & 90.93 & 91.79 \\
\hline FN-best-sem & 91.87 & 92.85 \\
\hline \hline TB2-basic & 85.75 & 87.47 \\
\hline TB2-best-no-www & 92.06 & 92.81 \\
\hline TB2-best & 92.92 & 93.62 \\
\hline \hline RRR-basic & 84.32 & 84.60 \\
\hline RRR-basic-mpf & 84.34 & 85.14 \\
\hline
\end{tabular}

Table 4: Results

$F N$-basic-flw uses v-surface, n1-surface, $\mathbf{p}$ and n2-surface on examples that follow the verb. $F N$ lex-syn- $f$ lw uses v-surface, v-pos, v-lemma, subcategorization, path (full POS), position, n1preposition, n1-surface, n1-pos, n1-lemma, n1parent, p, n2-surface, n2-pos, n2-lemma, n2det and parser-vote on examples that follow the verb. FN-best-no-sem uses v-surface, v-pos, vlemma, subcategorization, path (reduced POS), position, n1-preposition, n1-surface, n1-pos, n1lemma-mpf, n1-parent, p, n2-surface, n2-pos, n2-lemma-mpf, n2-det, parser-vote, count-ratio and pp-count on all examples. FN-best-sem uses the same set of features as $F N$-best-no-sem plus $\mathbf{v}$ frame and $\mathbf{n 1 - s r}$.

TB2-basic uses v-surface, n1-surface-mpf, $\mathbf{p}$ and n2-surface-mpf. TB2-best-no-www uses vsurface, v-pos, v-lemma, subcategorization, path (reduced POS), n1-preposition, n1-surface, n1mpf, n1-pos, n1-lemma, n1-np-label, p, n2surface, n2-mpf and n1-p-distance. TB2-best also uses count-ratio and pp-count.
RRR-basic uses v-surface, n1-surface, $\mathbf{p}$ and n2-surface. $\quad R R R$-basic-mpf uses v-surface, n1surface-mpf, $p$ and $\mathbf{n}$-surface-mpf.

On the FN dataset, all features except v-voice have a positive contribution to the system (n2-det, choice between semantic vs. thematic role and how should morphological processing be applied is questionable). The negative impact for the v-voice feature may be explained by the fact that the only situation in which it may potentially help is extremely rare: passive voice and the agent headed by "by" appears after another argument of the verb (i.e.: "The painting was presented to the audience by its author."). Moreover the PP-attachment based on the preposition "by" is not highly ambiguous; as seen in Table 3 in the FrameNet dataset, 88\% of the "by" ambiguity instances are verb-attachments.

The experiment with the highest cross-validation accuracy has an accuracy of $92.85 \%$ on the test data. The equivalent experiment that doesn't include manually annotated semantic information has an accuracy of $91.79 \%$ on the test data.

On TB2 dataset, the results are close to the results obtained on the FrameNet corpus, although the distribution of noun and verb attachment differs considerably between the two data sets $(70.28 \%$ are verbattachments in FN2 and $35.71 \%$ in TB2). The best accuracy in cross-validation is $92.92 \%$, which leads to an accuracy on test set of $93.62 \%$.

\section{Comparison with previous work}

Because we couldn't use the standard dataset used in PP-attachment resolution (Ratnaparkhi's), we implemented back-off algorithm developed by Collins and Brooks (1995) and applied it to our TB2 dataset. Both RRR and TB2 datasets are extracted from Penn Treebank. This algorithm, trained on TB2 training set, obtains an accuracy on TB2 test set of $86.1 \%$ (85.8\% when no morphological processing is applied). The same algorithm provides an accuracy on RRR dataset of $84.5 \%$ (84.1\% without morphological processing). The difference in accuracy between the two datasets is $1.6 \%$ (1.7\% without morphological processing when using Collins and Brooks's algorithm.

The difference in accuracy between a SVM model applied to RRR dataset (RRR-basic experiment) and the same experiment applied to TB2 dataset (TB2- 


\begin{tabular}{|l|c|l|l|}
\hline Description & Accuracy & Data & Extra Supervision \\
\hline \hline Always noun & 55.0 & RRR & \\
\hline Most likely for each P & 72.19 & RRR & \\
\hline Most likely for each P & $\mathbf{7 2 . 3 0}$ & TB2 & \\
\hline Most likely for each P & $\mathbf{8 1 . 7 3}$ & FN & \\
\hline Average human, headwords (Ratnaparkhi et al., 1994) & 88.2 & RRR & \\
\hline Average human, whole sentence (Ratnaparkhi et al., 1994) & 93.2 & RRR & \\
\hline \hline Maximum Likelihood-based (Hindle and Rooth, 1993) & 79.7 & AP & \\
\hline Maximum entropy, words (Ratnaparkhi et al., 1994) & 77.7 & RRR & \\
\hline Maximum entropy, words \& classes (Ratnaparkhi et al., 1994) & 81.6 & RRR & \\
\hline Decision trees (Ratnaparkhi et al., 1994) & 77.7 & RRR & \\
\hline Transformation-Based Learning (Brill and Resnik, 1994) & 81.8 & & WordNet \\
\hline Maximum-Likelihood based (Collins and Brooks, 1995) & 84.5 & RRR & \\
\hline Maximum-Likelihood based (Collins and Brooks, 1995) & $\mathbf{8 6 . 1}$ & TB2 & \\
\hline Decision trees \& WSD (Stetina and Nagao, 1997) & 88.1 & RRR & WordNet \\
\hline Memory-based Learning (Zavrel et al., 1997) & 84.4 & RRR & LexSpace \\
\hline Maximum entropy, unsupervised (Ratnaparkhi, 1998) & 81.9 & & \\
\hline Maximum entropy, supervised (Ratnaparkhi, 1998) & 83.7 & RRR & \\
\hline Neural Nets (Alegre et al., 1999) & 86.0 & RRR & WordNet \\
\hline Boosting (Abney et al., 1999) & 84.4 & RRR & \\
\hline Semi-probabilistic (Pantel and Lin, 2000) & 84.31 & RRR & \\
\hline Maximum entropy, ensemble (McLauchlan, 2001) & 85.5 & RRR & LSA \\
\hline SVM (Vanschoenwinkel and Manderick, 2003) & 84.8 & RRR & \\
\hline Nearest-neighbor (Zhao and Lin, 2004) & 86.5 & RRR & DWS \\
\hline \hline FN dataset, w/o semantic features (FN-best-no-sem) & $\mathbf{9 1 . 7 9}$ & FN & PR-WWW \\
\hline FN dataset, w/ semantic features (FN-best-sem) & $\mathbf{9 2 . 8 5}$ & FN & PR-WWW \\
\hline TB2 dataset, best feature set (TB2-best) & $\mathbf{9 3 . 6 2}$ & TB2 & PR-WWW \\
\hline
\end{tabular}

Table 5: Accuracy of PP-attachment ambiguity resolution (our results in bold)

basic experiment) is $2.9 \%$. Also, the baseline - the most probable PP type for each preposition - is approximately the same for the two datasets $(72.19 \%$ on RRR and $72.30 \%$ on TB2).

One may hypothesize that the majority of the algorithms for PP-attachment disambiguation obtain no more than $4 \%$ increase in accuracy on the TB2 compared to the results on the RRR dataset. One important difference between the two datasets is the size - 20,801 training examples in RRR vs. 54,629 training examples in TB2. We plan to implement more algorithms described in literature in order to verify this statement.

Table 5 summarizes the results in PP-attachment ambiguity resolution found in literature along with our best results.

Other acronyms used in this table:

- AP - dataset of 13 million word sample of Associated Press news stories from 1999 (Hindle and Rooth, 1993).

- LexSpace - Lexical Space - a method to measure the similarity of the words (Zavrel et al., 1997).
- LSA - Latent Semantic Analysis - measure the lexical preferences between a preposition and a noun or a verb (McLauchlan, 2001)

- DWS - Distributional Word Similarity. Words that tend to appear in the same contexts tend to have similar meanings (Zhao and Lin, 2004)

- PR-WWW - the probability ratio between verb-preposition-noun and noun-prepositionnoun constructs measured using World Wide Web searching.

\section{Conclusions}

The Penn Treebank-II results indicate that the new features used for the disambiguation of PPattachment provide a very substantial improvement in accuracy over the base line (from $87.48 \%$ to 93.62\%). This represents an absolute improvement of approximately $6.14 \%$, equivalent to a $49 \%$ error drop. The performance of the system on Penn Treebank-II exceeds the reported human expert performance on Penn Treebank-I (Ratnaparkhi et al., 1994) by about $0.4 \%$. A significant improvement comes from the unsupervised information collected 
from a very large corpus; this method proved to be efficient to overcome the data sparseness problem.

By analyzing the results from the FrameNet dataset, we conclude that the contribution of the gold semantic features (frame and semantic role) is significant $(1.05 \%$ difference in accuracy; $12.8 \%$ reduction in the error). We will further investigate this issue by replacing gold semantic information with automatically detected semantic information. Our additional lexico-syntactic features increase the accuracy of the system from $86.44 \%$ to $89.61 \%$ for PPs following the verb. This suggests that on the FrameNet dataset the proposed syntactic features have a considerable impact on the accuracy.

The best TB2 feature set is approximately the same as the best FN feature set in spite of the differences between the datasets (Parse trees: TB2 gold standard; FN - automatically generated. PPattachment ambiguity identification: TB2 - parse trees; FN - a combination of trees and FE annotation. Data source: TB2 - WSJ articles; FN - BNC). This fact suggests that the selected feature sets do not exploit particularities of the datasets and that the features are relevant to the PP-attachment ambiguity problem.

\section{References}

Steven Abney, Robert E. Schapire, and Yoram Singer. 1999. Boosting applied to tagging and PP Attachment. In Proceedings of EMNLP/VLC-99, pages 38-45.

Martha A. Alegre, Josep M. Sopena, and Agusti Lloberas. 1999. Pp-attachment: A committee machine approach. In Proceedings of EMNLP/VLC-99, pages 231-238.

Collin F. Baker, Charles J. Fillmore, and John B. Lowe. 1998. The Berkeley FrameNet Project. In Proceedings of the 17th international conference on Computational Linguistics, pages $86-90$.

Eric Brill and Philip Resnik. 1994. A rule-based approach to prepositional phrase attachment disambiguation. In Proceedings of the 15th conference on Computational Linguistics, pages 1198-1204.

Eugene Charniak. 2000. A Maximum-Entropy-Inspired Parser. In Proceedings of NAACL-2000, pages 132-139.

Michael Collins and James Brooks. 1995. Prepositional Phrase Attachment through a Backed-Off Model. In Proceedings of the Thirds Workshop on Very Large Corpora, pages 27-38.

Corinna Cortes and Vladimir Vapnik. 1995. Support-Vector Networks. Machine Learning, 20(3):273-297.
Daniel Gildea and Daniel Jurafsky. 2002. Automatic Labeling of Semantic Roles. Computational Linguistics, 28(3):245288.

Donald Hindle and Mats Rooth. 1993. Structural Ambiguity and Lexical Relations. Computational Linguistics, 19(1):103-120.

Mitchell Marcus, Beatrice Santorini, and Mary Ann Marcinkiewicz. 1993. Building a large annotated corpus of English: the Penn Treebank. Computational Linguistics, 19(2):313-330.

Mark McLauchlan. 2001. Maximum Entropy Models and Prepositional Phrase Ambiguity. Master's thesis, University of Edinburgh.

Marian G. Olteanu. 2004. Prepositional Phrase Attachment ambiguity resolution through a rich syntactic, lexical and semantic set of features applied in support vector machines learner. Master's thesis, University of Texas at Dallas.

Patrick Pantel and Dekang Lin. 2000. An unsupervised approach to Prepositional Phrase Attachment using contextually similar words. In Proceedings of the 38th Meeting of the Association for Computational Linguistic, pages 101-108.

Sameer Pradhan, Kadri Hacioglu, Wayne Ward, James H. Martin, and Daniel Jurafsky. 2003. Semantic Role Parsing: Adding Semantic Structure to Unstructured Text. In Proceedings of the International Conference on Data Mining, pages 629-632.

Adwait Ratnaparkhi, Jeff Reynar, and Salim Roukos. 1994. A Maximum Entropy Model for Prepositional Phrase Attachment. In Proceedings of the Human Language Technology Workshop, pages 250-255.

Adwait Ratnaparkhi. 1998. Statistical Models for Unsupervised Prepositional Phrase Attachment. In Proceedings of the 36th conference on Association for Computational Linguistics, pages 1079-1085.

Jiri Stetina and Makoto Nagao. 1997. Corpus based PP attachment ambiguity resolution with a semantic dictionary. In Proceedings of the Fifth Workshop on Very Large Corpora, pages 66-80.

Bram Vanschoenwinkel and Bernard Manderick. 2003. A weighted polynomial information gain kernel for resolving Prepositional Phrase attachment ambiguities with Support Vector Machines. In Proceedings of the Eighteenth International Joint Conference on Artificial Intelligence, pages 133-140.

Martin Volk. 2001. Exploiting the WWW as a corpus to resolve PP attachment ambiguities. In Proceedings of Corpus Linguistics, pages 601-606.

Jakub Zavrel, Walter Daelemans, and Jorn Veenstra. 1997. Resolving PP attachment Ambiguities with Memory-Based Learning. In Proceedings of CoNLL-97, pages 136-144.

Shaojun Zhao and Dekang Lin. 2004. A Nearest-Neighbor Method for Resolving PP-Attachment Ambiguity. In Proceedings of IJCNLP-04. 